\title{
Computer modeling of displacement cargo beam from cargo cart in system Mathcad
}

\author{
Evgenij Kudryavtsev ${ }^{1 *}$ \\ ${ }^{1}$ Moscow State University of Civil Engineering, Yaroslavskoye Shosse, 26, Moscow, 129337, Russia
}

\begin{abstract}
Article describe processes modeling of cargo beam displacements from cargo cart in system Mathcad $-\mathrm{u}(\mathrm{x})$, tilting angle $-\Theta(\mathrm{x})$, maximum sagging stresses $-\sigma \max (\mathrm{t})$ and internal efforts $(\mathrm{M}(\mathrm{x}), \mathrm{Q}(\mathrm{x}))$ at various positions of the cargo cart; algorithm and a complex of programs for automation of modeling and graphical representation of displacements and maximum sagging stresses in system Mathcad. The proposed procedures of modeling of displacements and maximum sagging stresses in any crosssection of a cargo beam allow to reduce time and cost for performance of such calculations at least in ten times and effectively to carry out researches when cargo beam parameters, cargo cart parameters and materials change.
\end{abstract}

\section{Introduction}

Computer modeling intended for decision of the diversified problems are receiving more and more propagation [1-13]. Wide use of computer modeling especially at the earliest stages of creation of products, processes and systems allow to reduce in many times huge losses at the next stages of cycle life.

The article describes process of computer modeling of displacements and maximum sagging stresses in various kinds: analytical; program and graphic in any cross-section of the cargo beam from moving of the cargo cart.

\section{Theoretical basis and computer modeling in system Mathcad}

Process of modeling of displacements and maximum sagging stresses in a cargo beam of the crane (the basic reactions, the displacements and maximum sagging stresses) on which the cargo cart (Figure 1) moves.

\footnotetext{
* Corresponding author: angela-1309.m@yandex.ru
} 


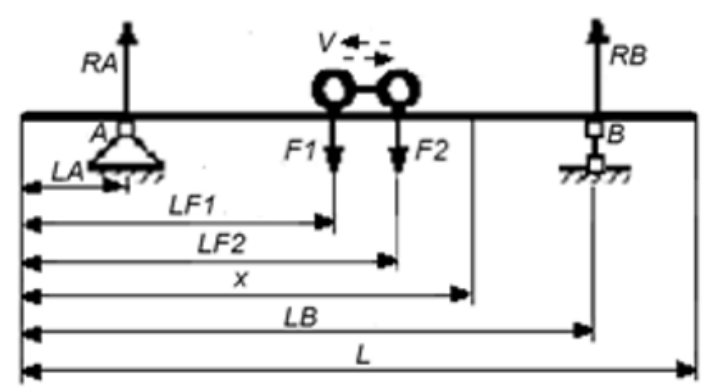

Fig. 1. Scheme to determination of displacements and maximum sagging stresses in a cargo beam of the crane from the cargo cart

Process of modeling of displacements and maximum sagging stresses includes some stages:

1. Full time - T, a step on time- $\Delta$ t and current time of movement of cargo cart with the first value for the built-in variable-FRAME equal 0 :

$$
T:=\frac{L-L t}{V} \quad \Delta t:=\frac{T}{P} \quad t:=F R A M E \cdot \Delta t
$$

where: $L$ - length of a cargo beam of the crane, $m$;

$L t$ - distance between axes of wheels of the cargo cart, $m$;

$V$ - speed of movement of the cargo cart, $\mathrm{km} / \mathrm{s}$;

$P$ - number of settlement positions of the cargo cart on a beam;

FRAME - built-in variable of animation with he first value equal 0 and the last - $P$.

2. Positions by 1 st and 2 nd supports of wheels of the cargo cart on a beam.

$$
L F 1(t):=V \cdot t ; \quad L F 2(t):=L F 1(t)+L t ;
$$

3. Analytical expressions for vertical reactions at supports $A$ and $B$ is defined from the sum of the moments (figure 1):

$$
\begin{aligned}
R B & :=\frac{F 1 \cdot L F 1(t)+F 2 \cdot L F 2(t)}{L} \\
R A(x) & :=\frac{F 1 \cdot(L-L F 1(t))+F 2 \cdot(L-L F 2(t))}{L}
\end{aligned}
$$

4. Programs of modeling of the bending moments - M(x) and cross-section forces $Q(x)$ in a cargo beam at various positions of the cargo cart $-x$ (Program 1):

$$
M(x):=\left|\begin{array}{l}
M R A \leftarrow R A \cdot(x-L A) \text { if } x \geq L A \\
M R B \leftarrow R B \cdot(x-L B) \text { if } x \geq L B \\
M F 1 \leftarrow F 1 \cdot(x-L F 1(t)) \text { if } x \geq L F 1(t) \\
M F 2 \leftarrow F 2 \cdot(x-L F 2(t)) \text { if } x \geq L F 2(t) \\
M \leftarrow M R A+M R B+M F 1+M F 2
\end{array} \quad Q(x):=\right| \begin{array}{ll}
Q R A \leftarrow R A \text { if } x \geq L A \\
Q R B \leftarrow R B \text { if } x \geq L B \\
Q F 1 \leftarrow F 1 \text { if } x \geq L F 1(t) \\
Q F 2 \leftarrow F 2 \text { if } x \geq L F 2(t) \\
Q \leftarrow Q R A+Q R B+Q F 1+Q F 2
\end{array}
$$

Fig. 2. Programs of modeling of the bending moments - $M(x)$ and cross-section forces - $Q(x)$ in a cargo beam at various positions of the cargo cart $-x$. 
5. Program of modeling of displacement of a cargo beam from moving of the cargo cart $-x$ (Program 2):

$$
E J u\left(x, u_{0}, \theta_{0}\right):=\mid \begin{aligned}
& E 0 \leftarrow E \cdot J \cdot u_{0}+E \cdot J \cdot \theta_{0} \cdot x \\
& E R A \leftarrow R A \cdot \frac{(x-L A)^{3}}{6} \text { if } x \geq L A \\
& E R B \leftarrow R B \cdot \frac{(x-L B)^{3}}{6} \text { if } x \geq L B \\
& E F 1 \leftarrow F 1 \cdot \frac{(x-L F I(t))^{3}}{6} \text { if } x \geq L F 1(t) \\
& E F 2 \leftarrow F 2 \cdot \frac{(x-L F 2(t))^{3}}{6} \text { if } x \geq L F 2(t) \\
& E \leftarrow E O+E R A+E R B+E F 1+E F 2
\end{aligned}
$$

Fig. 3. Program of modeling of displacement of a cargo beam from moving of the cargo cart $-x$

6. Analytic representations of displacements of the cargo beam $-u(x)$, tilting angle$\Theta(x)$ and maximum sagging stress of the cargo beam - $\operatorname{\sigma max}(t)$ :

$$
\begin{gathered}
u_{0}:=0 \cdot m \quad \theta_{0}:=0 \quad \text { Given } \quad \operatorname{EJu}\left(L A, u_{0}, \theta_{0}\right)=0 \quad \operatorname{EJu}\left(L B, u_{0}, \theta_{0}\right)=0 \\
\left(\begin{array}{l}
u_{0} \\
\theta_{0}
\end{array}\right):=\operatorname{Find}\left(u_{0}, \theta_{0}\right) \quad u_{0}=-0.016 \cdot m \quad \theta_{0}=0.487 \cdot \operatorname{deg} \quad u(x):=\frac{\operatorname{EJu}\left(x, u_{0}, \theta_{0}\right)}{E \cdot J} \\
\theta(x):=\frac{d}{d x} u(x) \\
M M(x):=|M(x)| \quad x:=L A \quad \text { Given } \quad 0 \cdot m \leq x \leq L \quad x_{\max }:=\operatorname{Maximize}(M M, x) \\
x_{\text {max }}=2 \cdot m \quad M M\left(x_{\max }\right)=1.2 \times 10^{5} J \quad \sigma_{\text {max }}(t):=\frac{M M\left(x_{\max }\right)}{W} \quad \sigma_{\text {max }}(t)=6 \times 10^{7} \mathrm{~Pa}
\end{gathered}
$$

7. Graphic representations of cargo beam displacements, $-u(x)$, tilting angle $-\Theta(x)$, maximum sagging stresses - $\sigma \max (t)$ and internal efforts $(M(x), Q(x))$ at various positions of the cargo cart:

$$
x:=0 \cdot m, \frac{L}{100} . . L \quad y(t):=0
$$

- initial value of parameter $F R A M E$ is equal 0 , section $p=0$ (Figure 2). 


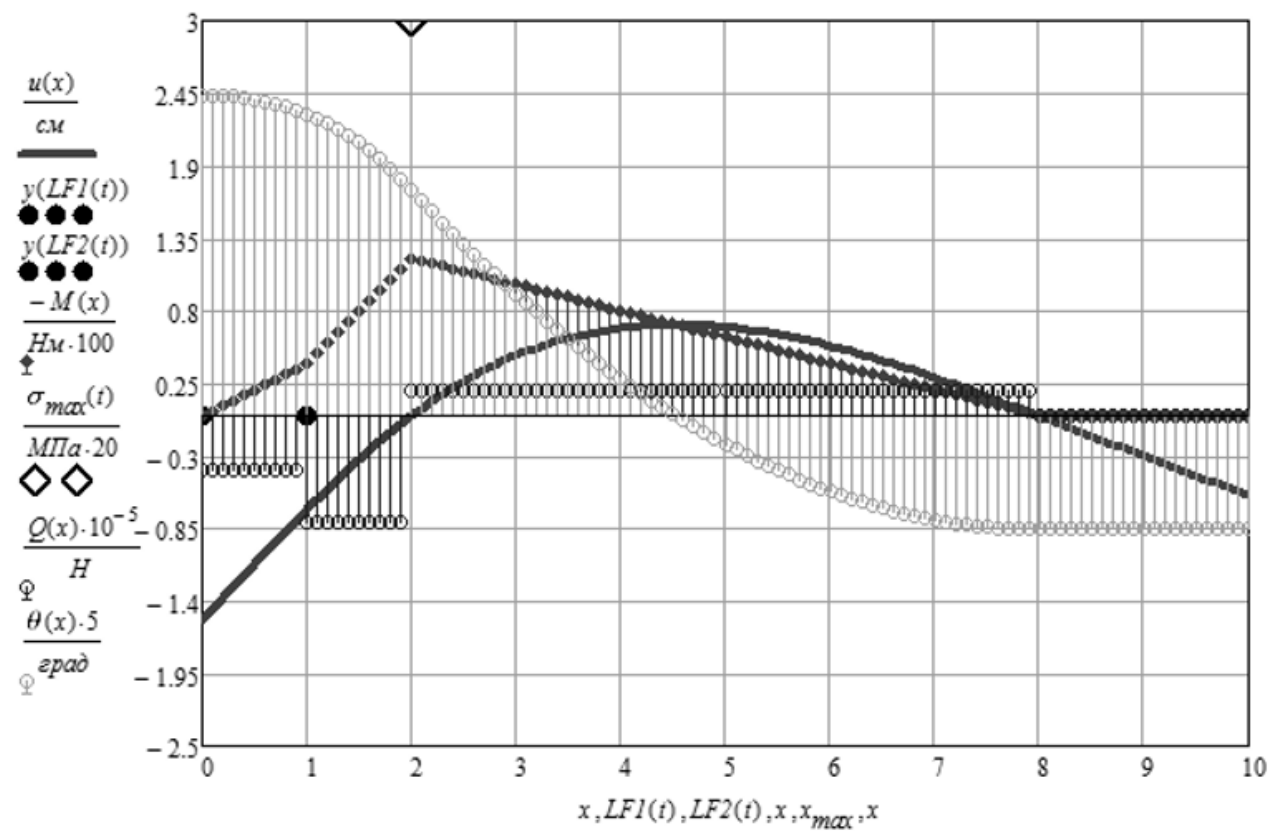

Fig. 4. Graphic representation of cargo beam displacements, the maximum sagging stresses and internal efforts at various positions $p$ of the cargo cart.

FRAME 1st (Figure 3).

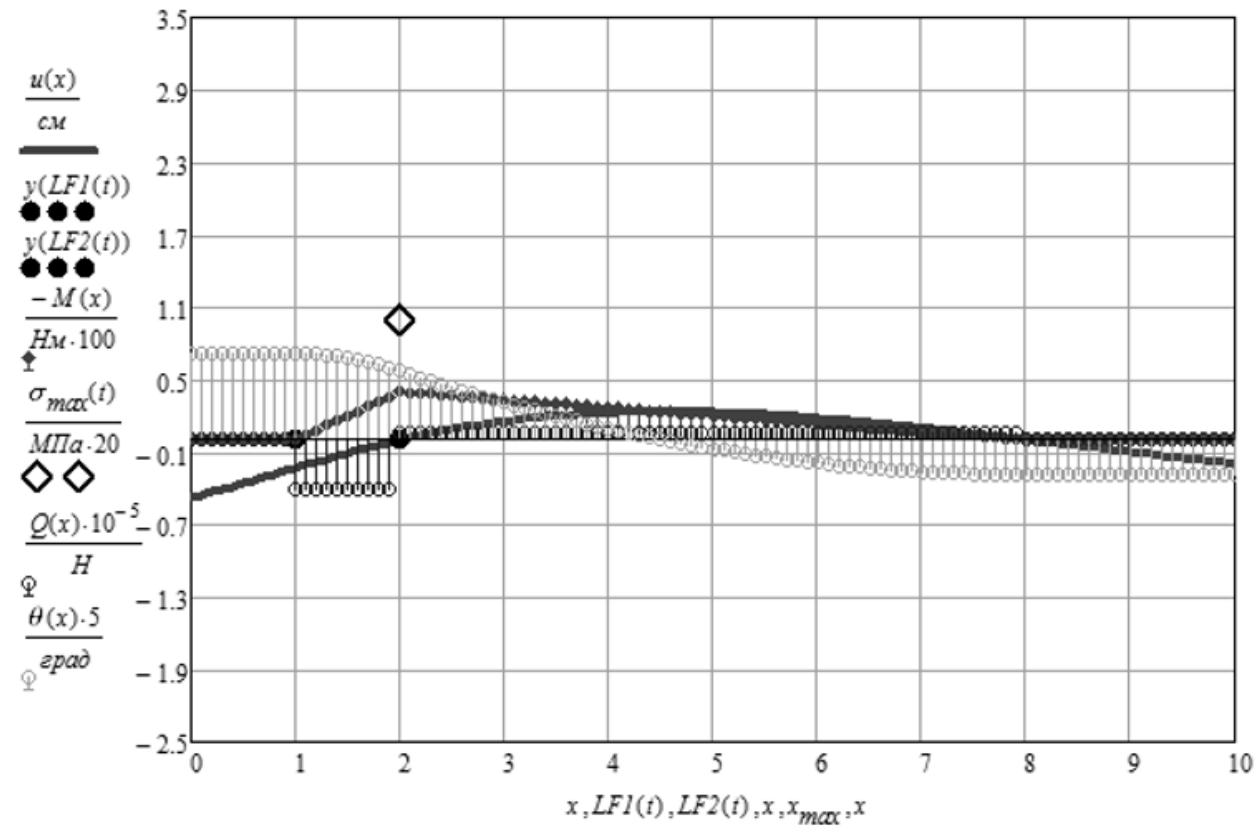

Fig. 5. Graphic representation of cargo beam displacements, the maximum sagging stresses and internal efforts at various positions $p$ of the cargo cart.

FRAME 2st (Figure 4). 


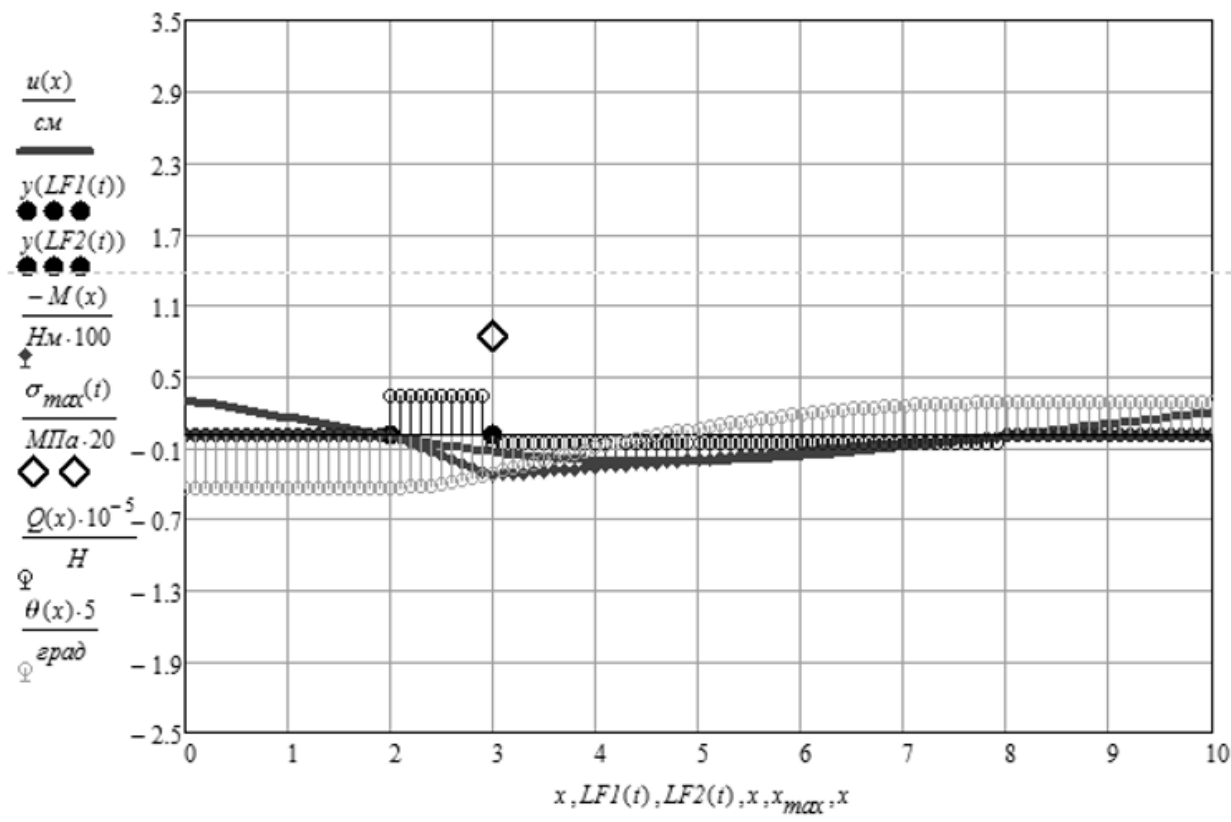

Fig. 6. Graphic representation of cargo beam displacements, the maximum sagging stresses and internal efforts at various positions $p$ of the cargo cart.

FRAME 3st (Figure 5).

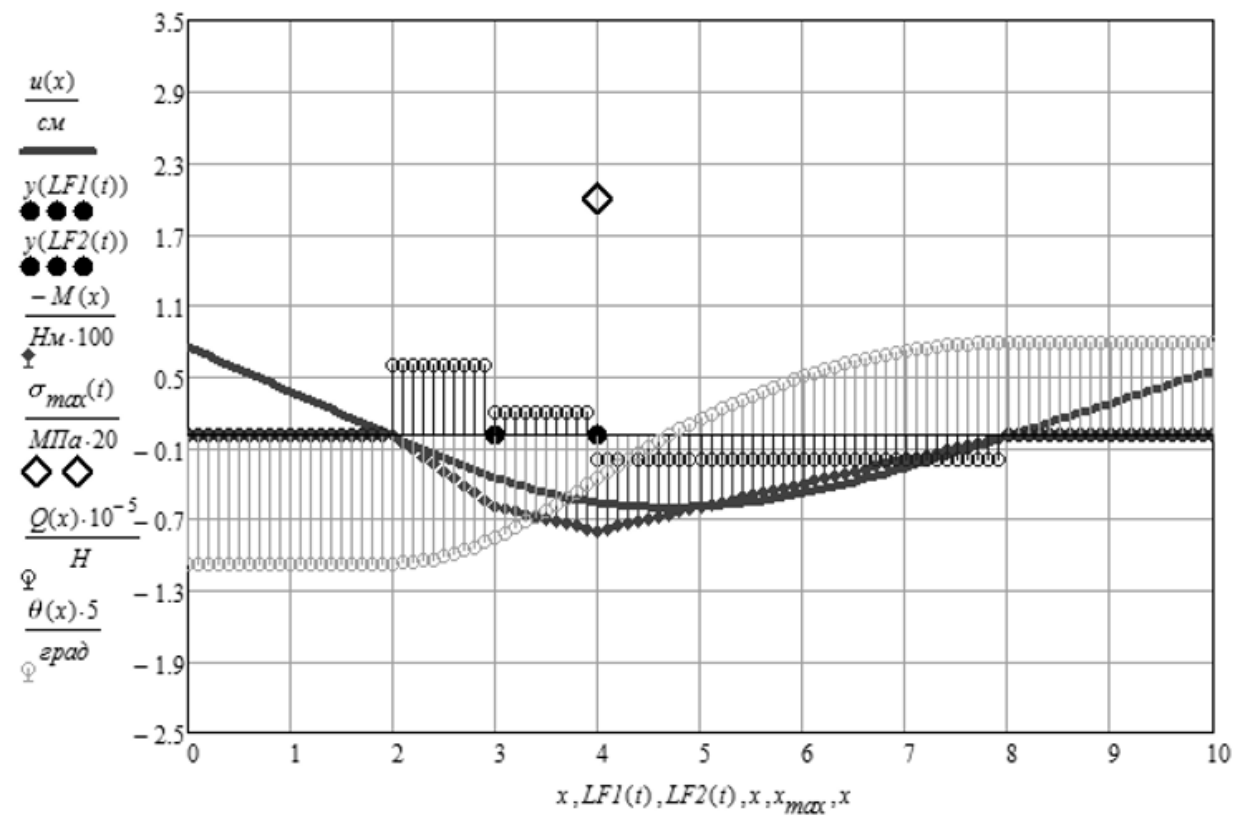

Fig. 7. Graphic representation of cargo beam displacements, the maximum sagging stresses and internal efforts at various positions $p$ of the cargo cart.

FRAME 4st (Figure 6). 


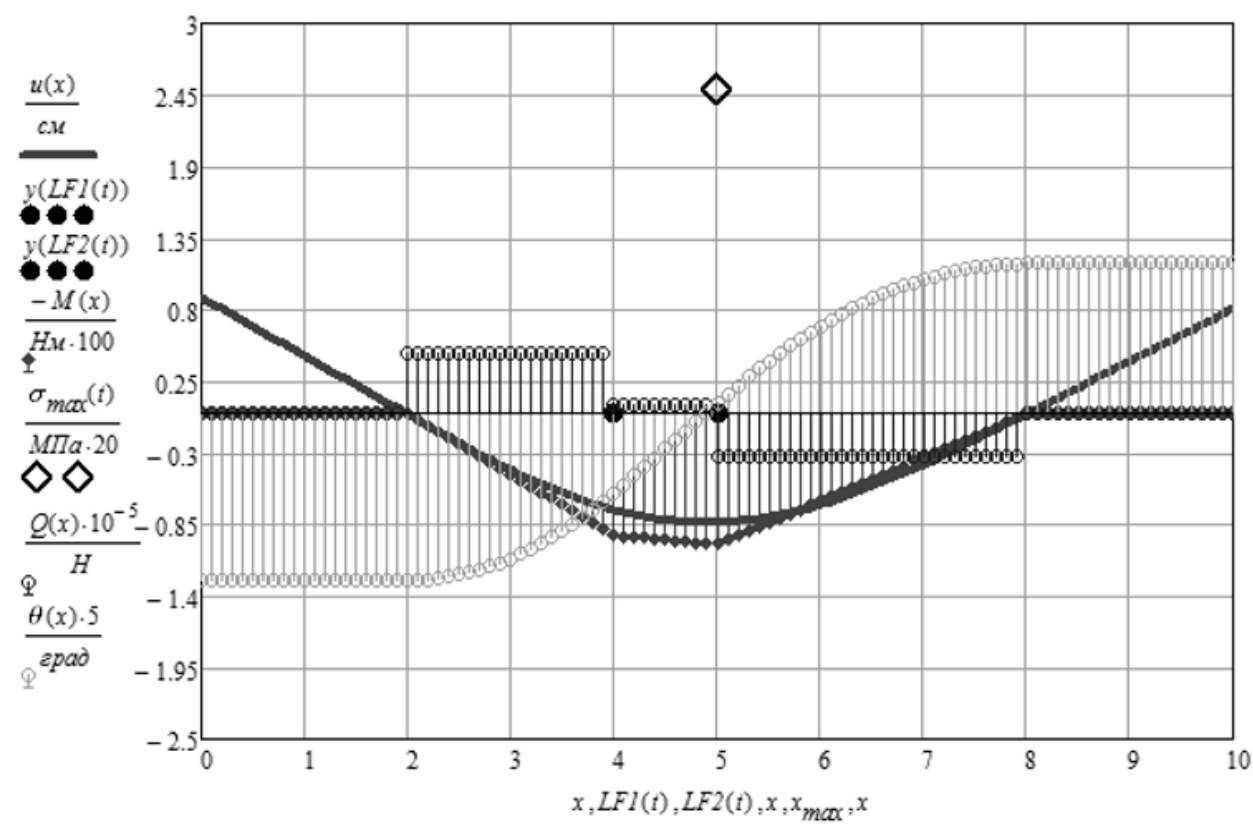

Fig. 8. Graphic representation of cargo beam displacements, the maximum sagging stresses and internal efforts at various positions $p$ of the cargo cart.

And so on. Following FRAMES of animation will be symmetric above resulted.

\section{Conclusion}

The main results of scientific research are created:

- analytical expressions of representation of cargo beam displacements, the maximum sagging stresses in any cargo beam cross-section depending on the positions of the cargo cart;

- algorithm and complex of programs of calculation and representation of cargo beam displacements, the maximum sagging stresses in any cargo beam cross-section in Mathcad system.

The proposed procedures of modeling of cargo beam displacements, the maximum sagging stresses in any cross-section of a cargo beam of the gantry crane from moving cargo cart allow to reduce time and cost for performance of such calculations at least in ten times and effectively to carry out researches when cargo beam parameters, cargo cart parameters and materials change.

\section{References}

1. T. Simankina, I. Kibireva, A.Mottaeva, M. Gusarova, Advances in Intelligent Systems and Computing. 983, 138-145, (2019) https://doi.org/10.1007/978-3-030-19868-8 13 
2. F. Beer and E. Jonston. Vector Mechanics for Engineers I-Static, II-Dynamics, (2011)

3. Shames, Engineering Mechanics: Statics and Dinamics, (2002).

4. R. Hibbeler, Engineering Mechanics: Principles of Statics and Dynamics, (2006)

5. R. Hibbeler, Structurial analises Prentice-Hall, (2006)

6. I. Karnovsky, O. Lebed, Advanced Methods of Structural Analysis, (2010)

7. L. Ushakov, A. Panichkin, World Transp. Techn. Machines, 50-54, (2014)

8. K. Leet, C. Uang. Fundamentals of Structural Analysis Tata McGraw-Hill, (2003)

9. J. Meriam, L. Kraige. Engineering Mechanics: I-Statics, II-Dinamics, (2008)

10. A, Williams. Structural analysis in theory and practice. Butterworth-Heinemann is an imprint of Elsevier, (2009)

11. T. Simankina, I. Kibireva, A.Mottaeva, M. Gusarova, Advan. Intell. Syst. Comput.. 983, 138-145 (2019) https://doi.org/10.1007/978-3-030-19868-8

12. L. Negi, R. Jangid. Structural Analysis Tata McGraw-Hill (2003)

13. R. R. Sharapov, V. S. Prokopenko, R. R. Sharapov, World Apl. Sci. J. 25(3),536542, (2015) 